\title{
MONITORING OF IMMUNE RESPONSE IN VIROLOGIC SUCCESSFULLY TREATED HIV-INFECTED PATIENTS IN SOUTHEASTERN SERBIA
}

\author{
Miodrag Vrbic, Maja Jovanovic, Lidija Popovic-Dragonjic, Aleksandar Rankovic, Marina Djordjevic-Spasic \\ Clinic for Infectious Diseases, Clinical Centre Niš, Serbia
}

\begin{abstract}
The number of CD4 lymphocytes defines the evolutional stage of HIV-infection and is the most important for a reliable estimation of the individual risk of developing AIDS. However, it is difficult to predict the degree of immune reconstitution during antiretroviral therapy, as it varies significantly from one person to another. Further investigations to better understand the limitations of immunological success are necessary to improve the response to treatment and regimen durability. The current study includes HIV-infected patients in Southeastern Serbia with achieved virologic suppression of HIV infection. The CD4 count was determined by flow cytometry, and was correlated with the duration of treatment, initial number of CD4 cells, type of antiretroviral therapy, mode of transmission of infection, age and gender of examinees. The resulting arithmetic mean and standard deviation of CD4 number was $473 \pm 259$ cells/ $\mu$ l (range, 1130 cells/ $\mu l$ ). There was no statistically significant correlation between the values of CD4 count and length of treatment, stage of the infection at which the therapy was started, treatment profile, method of infection, age or gender. The obtained results are comparable with the existing studies that follow immunological response to antiretroviral therapy and primarily point out the issue of substantial individual response variability, which has not yet been fully elucidated.
\end{abstract}

Key words: $H I V$, immune response, $c A R T$.

\section{Introduction}

During an HIV infection, the complex immunopathogenetic relationship of viral replication, destruction of infected CD4 lymphocytes, and immune response status passes through three phases. The primary infection phase is characterized by a massive viremia, drop of CD4 count, and induction of, primarily, virus-specific cytotoxic lymphocytes. After a period of several weeks up to several months, the parameters find a kind of balance among them and maintain it during the chronic phase of clinical latency. Its highly variable duration, from only a couple of years to even over 15 years, is characterized by progressive anatomical and functional damage to the lymphatic tissue. Eventually, the inability to maintain HIV-specific immune response results in a repeated viral load increase and drop of CD4 count, with the development of the acquired immune deficiency syndrome (AIDS), and presentations of opportunistic infections and tumors.

Combination antiretroviral therapy (cART) substantially reduces the incidence of AIDS and mortality, and now the World Health Organization consolidated guidelines that favor the use of HIV viral load (VL) monitoring for routine identification of its efficacy [1].

Correspondence to: Miodrag Vrbic, M.D., PhD

Clinic for Infectious Diseases, Clinical Centre Niš

48 Dr. Zoran Đinđić Blvd, 18000 Niš, Serbia

Phone: +381644890908

E-mail: mvrbic@open.telekom.rs

Received May $29^{\text {th }}, 2017$, accepted for publication June $6^{\text {th }}, 2017$
However, the monitoring of immunological response to treatment (defined as an increase in CD4 cell count) remains an important clinical tool, being significantly and independently associated with improved prognosis.

Two reference values are generally accepted: above 400-500 CD4 cells/ $\mu 1$, severe AIDS-related diseases are very rare; below $200 \mathrm{CD} 4 \mathrm{~T}$ cells/ $\mu$, the risk of AIDSrelated morbidity increases with increased duration of immunosuppression.

However, it is difficult to predict the degree of immune reconstitution, as it varies significantly from one person to another. Hence, the precise definition of the success of an immunological treatment does not exist at the moment, and depending on the study it is taken to be an increase of 50,100 or $150 \mathrm{CD} 4 \mathrm{~T}$ cells/ $\mu \mathrm{l}$ per year $[2,3]$.

In such a situation, further investigations to better understand the limitations of immunological success are necessary to improve individual responses to treatment and regimen durability.

\section{Material and Methods}

The study included patients on the cART, with achieved virologic suppression of HIV infection (HIV-VL $<50$ copies/ml) [4], in whom the number of CD4 cells was determined by flow cytometry (BD FACS Count ${ }^{\mathrm{TM}}$ ).

The patients in whom reduced CD4 lymphocyte counts could be the consequence of concomitant conditions, such as liver cirrhosis, autoimmune diseases, or 
immunosuppressive treatments, were excluded from the study.

Due to possible fluctuations in individual measurements, only those results were taken into account that were consistent with previous CD4 count control measurements, usually performed in six-month intervals.

The obtained results were displayed individually on the scatter diagram, in relation to the duration of treatment, and were also expressed by overall arithmetic mean with standard deviation. The significance of the correlation of their values with treatment duration, initial number of CD4 cells (stage of infection at which cART was started), type of cART, mode of transmission of infection, age and gender of patients, were determined by Student's t-test and a correlation coefficient $r$.

The study was conducted at the Clinic for Infectious Diseases - Clinical Center Niš, the reference center for HIV-infection treatment in the region of Southeastern Serbia.

\section{Results}

The study involved 58 patients, 44 (76\%) men and 14 (24\%) women, with the following age distribution: 12 (20\%) aged 20 to 30 years, $21(36 \%)$ aged 30 to 40 years, $13(23 \%)$ aged 40 to 50 years, $8(14 \%)$ aged 50 to 60 , and $4(7 \%)$ aged above 60 years of age.

In relation to the mode of transmission, $15(26 \%)$ examinees were infected via intravenous drug abuse, 26 (44\%) via homosexual intercourse (MSM), 12 (21\%) via heterosexual intercourse, $1(2 \%)$ via blood transfusion, while in $4(7 \%)$ patients the mode of transmission could not be established.

Combination ART was started in $40(69 \%)$ examinees with CD4 lymphocyte counts $\leq 200 / \mu 1$, and in 18 (31\%) examinees with CD4 counts $>200 / \mu 1$. In 28 $(48 \%)$ cases, it was based on protease inhibitors (PI), and in $30(52 \%)$ on non-nucleoside reverse transcriptase inhibitors (NNRTI).

Individual results of CD4 count measurements were correlated with the duration of cART, with the final presented value involving all the patients with $\geq 10$ years of treatment (Figure 1). The arithmetic mean with standard deviation of cART duration was 5.72 \pm 3.32 years.

The arithmetic mean with standard deviation of CD4 lymphocyte count was $473 \pm 259$ cells/ $\mu 1$, (range, 1130 cells $/ \mu 1$ ) (Figure 1). Most of the examinees $(33 ; 57 \%)$ had CD4 counts $\leq 500 / \mu 1$, while 25 (43\%) examinees had CD4 counts $\geq 500 / \mu 1$. Of the total number, $10(17 \%)$ examinees had $\leq 200 \mathrm{CD} 4$ cells $/ \mu 1,3$ out of 8 patients $(37 \%)$ at the beginning of treatment (in the first cART year), and $7(14 \%)$ out of the remaining 50 examinees after $\geq 3$ years of treatment.

The association of CD4 cell counts per $\mu l$ with cART duration was not statistically significant. There was not any statistically significant difference in CD4 counts related to the initial number of CD4 cells $(p>$ $0,05)$, type of cART, mode of transmission of infection, age and gender of patients.

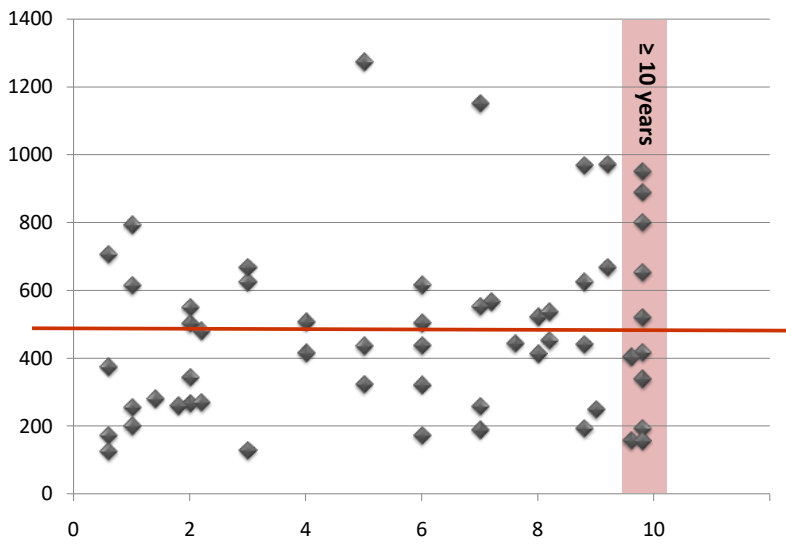

Fig. 1 Individual counts of CD4 cells/ $\mu \mathrm{l}$ (y - axis) related to the duration of ART in years ( $\mathrm{x}$ - axis), with arithmetic mean of CD4 counts in cells $/ \mu 1$.

\section{Discussion}

The average number of 467 CD 4 cells/ $\mu 1$ obtained in the study and absence of complete recovery of the immune system in most of the patients $(43 \%$ examinees $\geq 500$ cells $/ \mu 1,57 \%$ examinees $<500$ cells $/ \mu \mathrm{l})$ corresponds to the results presented in numerous studies of immune reconstitution after multiannual antiretroviral therapy followed by a complete virologic response $[5,6,7,8]$.

Since CD4 counts after several years of treatment achieve a plateau, characterized by small or absent increases, the connection between these two parameters was not evaluated in the above studies., The present study, on the other hand, included patients at the beginning of cART, when the dynamics of change in CD4 lymphocyte counts was most conspicuous - usually characterized by a rapid increase in the number of circulating CD4 cell lymphocytes during the first 2-3 months of treatment (median of 21.2 cells $/ \mu 1$ per month), representing mainly a redistribution of activated CD4 memory cells previously sequestered in the lymphoid tissue and generalized reduction in apoptotic cell death, followed by a slower second phase of CD4 cell expansion (5.5 cells/ $\mu$ l per month), representing the expansion of naive CD4 cells [9].

However, that did not produce any significant change in the overall study results, nor did it reveal any significant association of CD4 lymphocytes with treatment duration. A possible explanation could be, according to recent recommendations, an earlier initiation of treatment (regardless of CD4 count), and now a reduced number of presentations with advanced HIV disease ( 3 of the 8 patients, i.e. $37 \%$ ). A smaller percentage of late presenters has also been reported in different European and US studies $[10,11,12]$.

The remaining 7 out of 50 examinees in whom CD4 counts were below $200 / \mu$ l after multiannual treatments, belonged to the group of immunological non-responders. Their prevalence of $14 \%$ was comparable with the results of other studies of such a discordant immune response, where satisfactory immune status could not be 
achieved despite prolonged viral suppression [13]. The consequences of this condition in the long run are not yet clear $[14,15]$. The data from the relevant clinical surveys suggest that mortality seems to be slightly higher, but has not been related to AIDS-defining diseases, the increased incidence of which is present only in the first few months of therapy [16]. The exact background of this discordant response is also unclear, and DHHS guidelines do not recommend switching an otherwise suppressive cART regimen in this patient group [17].

In general, the risk factors for the lack of immunologic response are heterogenous and often unmodifiable. Firstly, a complete reconstitution of the immune system is only rarely possible if the patient's initial situation is poor (the worse the immune system, the more unlikely a complete recovery), and viral suppression over several years cannot change that $[6,8,18]$. Forty-four percent of patients with less than $100 \mathrm{CD} 4$ cells/ $\mu$ at the initiation of cART failed to reach $500 \mathrm{CD} 4$ cells $/ \mu$ l even after a decade of virologic successful treatment, while $25 \%$ of patients with $100-200$ CD4 cells $/ \mu$ still showed that risk $[14,19]$. The trials of several thousand patients confirmed a connection of the initial CD4 count with the level of an immune response, as suggested by their correlation as well at the borderline of statistical significance $(p>0.05)$, which was verified by the presented study of a much smaller number of subjects.

Age may also play a role. In older patients, immunological response is often only moderate, mainly due to thymic degeneration [20,21]. This observation has not been confirmed by the present study either, probably

\section{References}

1. Consolidated guidelines on the use of antiretroviral drugs for treating and preventing HIV infection: Monitoring the response to ART and the diagnosis of treatment. World Health Organization 2013; 7:133-137.

2. Migueles SA, Connors M. Success and failure of the cellular immune response against HIV-1. Nature Immunology 2015; 16:563-570.

3. EACS Guidelines Version 8.1 ostober 2016 /EACS Guidelines 8.1 PART II 11.

4. Gatell JM, Rockstroh JK, Furrer H, et al. EACS Guidelines Version 8.2 january 2017 PART II 12

5. Mocroft A, Phillips AN, Gatell J, et al. Normalisation of CD4 counts in patients with HIV-1 infection and maximum virological suppression who are taking combination antiretroviral therapy: an observational cohort study. Lancet 2007; 370:407-413.

6. Kaufman GR, Furrer H, Ledergerber B, et al. Characteristics, determinants, and clinical relevance of CD4 $\mathrm{T}$ cell recovery to < 500cells/microL in HIV type 1-infected individuals receiving potent antiretroviral therapy. Clin Infect Dis 2005; 41:362-372.

7. Moing V, Thiebaut R, Chene G, et al. Long-term evolution of CD4 count in patients with a plasma HIV RNA persistently $<500$ copies $/ \mathrm{mL}$ during treatment with antiretroviral drugs. HIV Medicine 2007; 8:156-163.

8. Bishop JD, DeShields S, Cunningham T, et al. CD4 Count Recovery After Initiation of Antiretroviral Therapy in Patients Infected With Human Immunodeficiency Virus. American Journal of the Medical Science 2016; 352:239-244.

9. Le Moing V, Thiebaut R, Chene G, et al. Predictors of log-term increase in CD4+ cell counts in HIV-infected patients receiving a protease inhibitor-containing antiretroviral regimen. J Infect Dis 2002; 185:471-480. due to the same reasons as for the impact of low number of initial CD4 cells. On the other hand, these results in a small number of patients indicate a substantial individual variability of the above-mentioned risk factors for poor immune response.

Other reasons that could possibly explain the wide range of immune responses $(1130$ cells/ $\mu 1$ in the present study), such as gender, mode of transmission of infection, or the difference between NNRTIs- and PI-based cART, were not associated with CD4 counts in the current study, as confirmed in other studies in the past $[22,23]$.

\section{Conclusion}

Alongside viral load, measurement of the CD4 cells level is the most important parameter or surrogate marker in HIV medicine. As the strongest predictor of HIV progression, allowing for a reliable estimation of the individual risk of developing AIDS, it is of vital importance for the patients.

Having in mind that variability of CD4 expression among patients is still completely unresolved, further efforts to improve the understanding of the success and failure of an immune response would have implications not only for HIV infection, but could also possibly help in the interpretation of the role of cellular immunity in other diseases.

10. Antinori A, Coenen T, Costagiola D, et al. Late presentation of HIV infection: a consensus definition. HIV Med 2011; 12:61-64.

11. Borghi V, Girardi E, Bellelli S, et al. Late presenters in an HIV surveillance system in Italy during the period 1992-2006. J Acquir Immune Defic Syndr 2008; 49:282-286.

12. Hoffmann C. Incidence and risk factors of late HIV diagnosis. In: Hoffmann C, Rockstroh JK, editors. HIV 2015/2016. Medizin Fokus Werlag, Hamburg 2015; 171-172.

13. Hoffmann C. Discordinant response. In: Hoffmann C, Rockstroh JK, editors. HIV 2015/2016. Medizin Fokus Werlag, Hamburg $2015 ; 148-150$

14. Kelley CF, Kitchen CM, Hunt PW, et al. Incomplete peripheral CD4+ cell count restoration in HIV-infected patients receiving long-term antiretroviral treatment. Clin Infect Dis. 2009; 48:787-794

15. Gazzola L, Tincati C,Monforte AA, Marchetti G. The absence of CD4+ $\mathrm{T}$ cell count recovery despite receipt of virologically suppressive highly active antiretroviral therapy: clinical risk, immunological gaps, and therapeutic options. Clin Infect Dis 2009; 48:328-337.

16. Zoufaly A, der Heiden M, Kollan C, et al. Clinical outcome of HIVinfected patients with discordant virological and immunological response to antiretroviral therapy. J Infect Dis 2011; 203:364-371.

17. Guidelines for the Use of Antiretroviral Agents in HIV-1-infected Adults and Adolescents. US Department of Healt and Human Services 2015-04-08.

18. Robbins GK, Spritzler JG, Chan ES, et al. Incomplete reconstitution of $\mathrm{T}$ cell subset on combination antiretroviral therapy in the AIDS Clinical Trials Group protocol 384. Clin Infect Dis 2009; 48:350-361. 
19. Lok JJ, Bosch RJ, Benson CA, et al. Long=term increase in CD4+ T-cell count during combination antiretroviral therapy for HIV-1 infection. AIDS 2010; 24:1867-1876.

20. Viard JP, Macroft A, Chiesi A, et al. Influenece of age on CD4 cell recovery in HIV-infected patients reciving HAART: evidence from EuroSIDA study. J Infect Dis 2001; 183:1290-1294.

21. Graber S, Kausignian I, Sobel A. at al. Immunologic and clinical responses to highly active antiretroviral therapy over 50 years of age. Reults from the French Hospital Database on HIV. AIDS 2004; 18:2029-2038.
22. Torti C, d'Armino-Monforte A, Pozniak AL, et al. Long-term CD4+ T-cell countevolution after switching from regimens including HIV nucleoside reverse transcriptase inhibitors (NRTI) plus protease inhibitors to regimens containing NRTI plus nonNRTI or only NRTI. BMC Infect Dis 2011:11-23.

23. Castilho JL, Melekhin VV, Sterlin TR. Sex Differences in HIV Outcomes in the Highly Active Antiretroviral Therapy Era: A Systematic Review. Res Hum Retroviruses 2014; 30:446-456. 